\title{
Symptomatic outcome following laparoscopic cholecystectomy
}

\author{
R. $\operatorname{Hasan}^{1}$, V. Abeysuriya ${ }^{2}$, S. Kumarage ${ }^{3}$, J.A.A.S. Wijesinghe ${ }^{4}$ \\ ${ }^{I}$ Department Of Anatomy, Faculty Of Medicine, University Of Kelaniya, Sri Lanka \\ ${ }^{2}$ Department Of Anatomy, Faculty Of Medicine, University Of Kelaniya, Sri Lanka \\ ${ }^{3}$ Department Of Surgery, Faculty Of Medicine, University Of Kelaniya, Sri Lanka \\ ${ }^{4}$ Faculty Of Medicine, University Of Kelaniya, Sri Lanka
}

\begin{abstract}
Introduction: The symptomatic outcome following laparoscopic cholecystectomy in patients with gallstone disease has notbeen evaluated extensively.

Objective:To assess the symptom prevalence of pre and postlaparoscopic cholecystectomy for symptomatic gallstone disease.

Methods: A descriptive study was carried out on 60 patients treated by laparoscopic cholecystectomyfrom 2013 to 2016.Patients with choledocholithiasis were excluded.The symptom profile of the patients was evaluated by a standardized interviewer administered questionnaire which included demographic details; pre and post-operative symptoms; returning to full activity; wound complications and their general acknowledgement of the surgical intervention. The questionnaire was administered to the patients at least three monthspostoperatively. To establish the relevance of abdominal symptoms to gallstone disease, the frequency of pre and post-operative symptoms were compared.

Results: Symptoms relieved by cholecystectomy were nausea, vomiting, colicky abdominal pain and back-pain. Flatulence, fat intolerance, and nagging abdominal pain were unaffected. Relief of heartburn outweighed the de novo development of this symptom after cholecystectomy.Post cholecystectomydiarrhoea occurred in 11\%. In the elderly age group (>60 years), significant number of patients regained full activity after laparoscopic cholecystectomy. Patient acknowledgementof a satisfactory cosmetic resultis $100 \%$ following the laparoscopic cholecystectomy.

Conclusion: Despite the persistence or de novo occurrence of symptoms, majority of patientsconsidered that they obtained symptomatic improvement following surgical treatment and were pleased with the end result.
\end{abstract}

\section{Introduction}

It has been observed that a considerable number of patients, continue to experience symptomseven after cholecystectomy. Some of these symptoms arise de novo after surgery. Loss of reservoir function of the gall bladder, which results in disturbance of bile metabolism and entero-hepatic cycling has been implicated as a cause. $^{1,2}$.This study was designed to assess symptomprevalenceof patients with symptomatic gallstone disease during pre and postlaparoscopic cholecystectomy.

\section{Methodology}

A descriptive study was carried out on 60 patients who underwent laparoscopic cholecystectomy during their pre and post-operative period during 2013 - 2016. Patients with choledocholithiasis were excluded.The patients were recruited from the surgical unit of the North Colombo Teaching Hospital. Ethical consent was obtained from the Ethical ReviewCommittee, Faculty of Medicine, University of Kelaniya,Sri Lanka.For statistical analysis, $t$ test was used. Differences were considered significant at $p<0.05$. The symptom profile of the patients was evaluated by a standardized interviewer administered questionnaire which include demographic details, pre-operative symptoms, postoperative symptomatic improvement; returning to full activity, surgical outcome and general appreciation of the surgical intervention. The questionnaire was administered to the patients three monthspostoperatively.In an attempt at establishing the relevance of abdominal symptoms to gallstone disease, the frequency of pre and post-operative symptoms of laparoscopic cholecystectomy were compared. For each symptom, the number of patients who were free of the complaint three or more months after operation was divided by the number of patients exhibiting the symptom preoperatively.

\section{Results}

Median age for the60 patients treated by laparoscopic cholecystectomywas 36.5years (range 22-76years). With regards to the abdominal symptoms and signs experienced by the patients preoperatively, most frequent being fatty food intolerance and heartburn, and nagging abdominal pain and nausea (Table 01) 
Table 02 shows the data on pre and postoperative abdominal symptoms. This show that the nausea, vomiting, colicky abdominal pain, and back pain are specific to gallstone disease whereas flatulence, fat intolerance, and nagging abdominal pain are not. Heartburn is probably related and the number of patients who were relieved of this symptom by cholecystectomy $(n=31)$ outweighed the number who developed it after cholecystectomy $(n=7)$. Overall, postoperative pain (including heartburn) was seen 37 laparoscopic cholecystectomized patients and 11 of these considered that their pain was the same as they had experienced before operation. In addition, 7 patients with pain were taking analgesics or antacid drugs on a regular basis.

Despite persistence of symptoms or their de novo occurrence, $98 \%$ who had a laparoscopic cholecystectomy experienced overall symptomatic improvement postoperatively. Furthermore 58 patients were pleased with the end result of the operation.Wound healing problems occurred in 4patients. 100\% of patients were pleased with their scar treated by laparoscopic cholecystectomy. Postoperative scar related pain was present in 5 .

None of the patients developed jaundice postoperatively, although 05 of them developed itching while anotherfour experienced shivering postoperatively.Post cholecystectomydiarrhoea occurred in 5 in the laparoscopic cholecystectomy group. After surgery, 3 patients developedsteatorrhoea, Regardless of sex, patients who had a laparoscopic cholecystectomy returned to workin medianof two weeks.

\section{Discussion}

This study has confirmed that patients with symptomatic gallstone disease exhibit a wide spectrum of symptoms, some of which are not relieved by cholecystectomy. It revealed nausea, vomiting, colicky abdominal pain, back pain, and heartburn are related to gallstone disease and in contrast fat intolerance, flatulent dyspepsia, and nagging abdominal pain do not seem to be related to the disease and are not influenced by cholecystectomy. These results are in keeping with the findings of previous reports that a large percentage of patients with gallstone disease have functional motility disturbances of the gastrointestinal tract, the symptoms of which are not changed by cholecystectomy. One previous study showed that socio-psychological factors seem to play an important role in the development of post cholecystectomy dyspepsia ${ }^{3}$.

The frequent presence of heartburn before cholecystectomy and its relief by this surgical treatment in most patients suggests a causal relationship between reflux disease and gallstones. The disappearance of heartburn postoperatively may be a reflection, however, of the natural history of gastro-oesophageal reflux disease. In this study, seven patients developed this symptom for the first time after cholecystectomy. Although this may be because of a change in patients' dietary habits after cholecystectomy, there is evidence that the higher oesophageal acid exposure in patients with cholelithiasis may be aggravated by cholecystectomy as a result of a fall in the lower oesophageal sphincter pressure ${ }^{4}$. The results of this symptomatic survey do not confirm this reported finding as symptomatic relief of heartburn by cholecystectomy outweighed its de novo occurrence postoperatively.

Post cholecystectomydiarrhoea was reported by $11 \%$ of the patients.A similar incidence has been reported previously ${ }^{4}$. Loose stools, with relapsing bouts of more abundant watery diarrhoea after removal of the gall bladdercan be explained by postoperative disturbance of bile metabolism and changes in the dynamics of bile release resulting in subclinical fat malabsorption ${ }^{5}$. Jaundice after cholecystectomy was not encountered in this study. However,literature revealed that an incidence of retained or missed common bile duct stones of $1 \%$ or less ${ }^{3,4}$.

Wound healing problems were reported by $9 \%$.This low infection rate relates to the state of the wound during the hospital period and ignores wound problems that are experienced by $60 \%$ of patients after discharge from hospital. Previous studies have shown that positive cultures of bile and gall bladder wall are associated with an increased incidence of wound infection. In laparoscopic cholecystectomy, the gall bladder is extracted through a small incision with frequent spilling of bile or stone fragments in the wound. Infection in the wound where the gall bladder is extracted is thus likely. In addition, the infra-umbilical wound is close to the umbilicus to permit the spread of organisms from the umbilicus.

In agreement with previous studies, this study confirmed that laparoscopic cholecystectomy is associated with a quicker postoperative recovery and a faster return to work or full activity. In addition, we have found that significantly more elderly patients (>60 years) have regained full activity at six months when the cholecystectomy is performed by the laparoscopic approach. This has important implications for community care of the elderly ${ }^{6}$.

By contrast with one previous report ${ }^{2,6} 93 \%$ of the patients who responded to the survey were pleased with the end result of the procedureand despite the frequent persistence of abdominal symptoms. A similar frequency of overall improvement despite a high number of patients with persistent abdominal pain post cholecystectomy has been reported previously ${ }^{4}$. The same study suggested that patients with biliary colic might have a better symptomatic outlook than those with more prolonged attacks of pain. This is confirmed in this study with a higher benefit ratio for colicky pain by comparison with nagging abdominal pain.

\section{Conclusion}

This study confirmed that laparoscopic cholecystectomy is associated with a quicker postoperative recovery and a faster return to work or full activity. Despite the persistence or de novo occurrence of symptoms, majority of 
patientsconsidered that they had obtained symptomatic improvement by their surgical treatment and were pleased with the end result.

\section{References}

[1]. Walsh TN, Russell RCG. Cholecystectomy and gallbladder conservation. BrJSurg. 1992; 79:4-5

[2]. Morgenstem L, Wong L, Berci G. Twelve hundred open cholecystectomies before the laparoscopic era. Arch Surg1992; 127: 400-3

[3]. Henry ML, Carey LC. Complications of cholecystectomy. SurgClin North Am.1983; 63:1191-204

[4]. Soper NJ, Flye MW, Brunt LM. Dark lining of silver cloud: Biliary complications of laparoscopic cholecystectomy. Gastroenterology 1992;38: A572

[5]. Hess Thaysen E, Pedersen L. Idiopathic bile acid catharsis. Gut1976; 17:965-70

[6]. VinueGC,Collet D, Larson GM,Cheadle WG, Miller FB, Perissat J. Interruption of professional and home activityafter laparoscopic cholecystectomy among French and American patients.Am Sure.1991; 161:396-8

Table 01: The abdominal symptoms and signs experienced by the patients preoperatively $(\mathrm{n}=60)$

\begin{tabular}{|l|l|}
\hline Preoperative symptoms & Number of patients \\
\hline Nagging abdominal pain & 33 \\
\hline Colicky abdominal pain & 28 \\
\hline Heartburn & 31 \\
\hline Vomiting & 22 \\
\hline Back pain & 27 \\
\hline Nausea & 18 \\
\hline Fatty food intolerance & 26 \\
\hline Flatulence & 21 \\
\hline Jaundice & 08 \\
\hline Fever & 09 \\
\hline Other & 05 \\
\hline
\end{tabular}

Table 02: Frequency of symptomatic relief $(n=60)$

\begin{tabular}{|l|l|l|l|}
\hline Symptom & Pre-operative occurrence & $\begin{array}{l}\text { Post-operative } \\
\text { occurrence }\end{array}$ & De novo \\
\hline Nausea & 18 & 02 & 01 \\
\hline Vomiting & 22 & 03 & 01 \\
\hline Flatulence & 21 & 08 & 04 \\
\hline Fat intolerance & 26 & 16 & 06 \\
\hline Heart burn & 31 & 07 & 03 \\
\hline Nagging pain & 33 & 10 & 05 \\
\hline Colicky pain & 28 & 06 & 04 \\
\hline Back pain & 27 & 02 & 01 \\
\hline
\end{tabular}

